\title{
Quantum Noise Limited Trace Gas Cavity Enhanced Polarization Spectroscopy
}

\author{
Ya J. Guan', Jiahao Dong1, Chathura P. Bandutunga' ${ }^{1}$, Roland Fleddermann', Timothy T-Y Lam ${ }^{1}$, Malcolm \\ B. Gray ${ }^{2}$ and Jong H. Chow ${ }^{1}$ \\ ${ }^{1}$ Research School of Physics and Engineering, Australian National University, Canberra, ACT 2601, AUSTRALIA \\ ${ }^{2}$ National Measurement Institute, Lindfield, NSW 2070, AUSTRALIA \\ E-mail: Yajie.Guan@anu.edu.au
}

\begin{abstract}
We present a new molecular trace gas absorption spectroscopy architecture and its recent progress. It uses optical cavity polarization states for an ultra-sensitive absorption readout, and achieves shot noise limited performance.

OCIS codes: (300.6320) Spectroscopy, high-resolution; (300.6360) Spectroscopy, laser; (300.6390) Spectroscopy, molecular; (140.4780) Optical resonators.
\end{abstract}

\section{Introduction}

Cavity enhanced laser absorption spectroscopy techniques have been developed and applied to a broad range of industrial and research applications [1]. Due to the use of an optical cavity, the light-gas interaction length can be resonantly enhanced resulting in enhanced sensitivity for gas concentration measurements, pressure detection and other metrology measurements [2]. Here we present a recently developed spectroscopic method that uses Polarization Impedance Matching Spectroscopy (PIMS) to manipulate and measure intra-cavity absorption. This novel technique is immune to relative intensity noise, enables a zero background DC readout, and can be laser modulation free. We will present results that demonstrate it approaches the system fundamental shot noise limit.

\section{Conceptual setup and Experiment}

For the PIMS system, the intra-cavity absorption is inferred from the impedance matching state [3] of an optical resonator and the readout is generated by the polarization properties of a non-degenerate, birefringent cavity. For such cavities, the two orthogonally polarized light ( $\mathrm{P}$ mode and $\mathrm{S}$ mode) resonate at well separated frequencies. The conceptual idea of PIMS is shown in Figure 1. The initial input optical field consisting of both S and P states (Fig. 1a) interrogates the cavity. When one of the polarizations is resonant (assuming $S$ state in Figure 1), it is able to interact with the absorbing medium and probes the intra-cavity absorption $(\alpha \mathrm{L})$. Conversely, the other orthogonal component (P state) is non-resonant and will be reflected immediately with no absorption. It is therefore used as a reference field. As a result, the reflected light of the cavity represented by the polarization diagram comprises both $\mathrm{S}$ mode as the probe and P mode as the reference field, as shown in Fig. 1b.

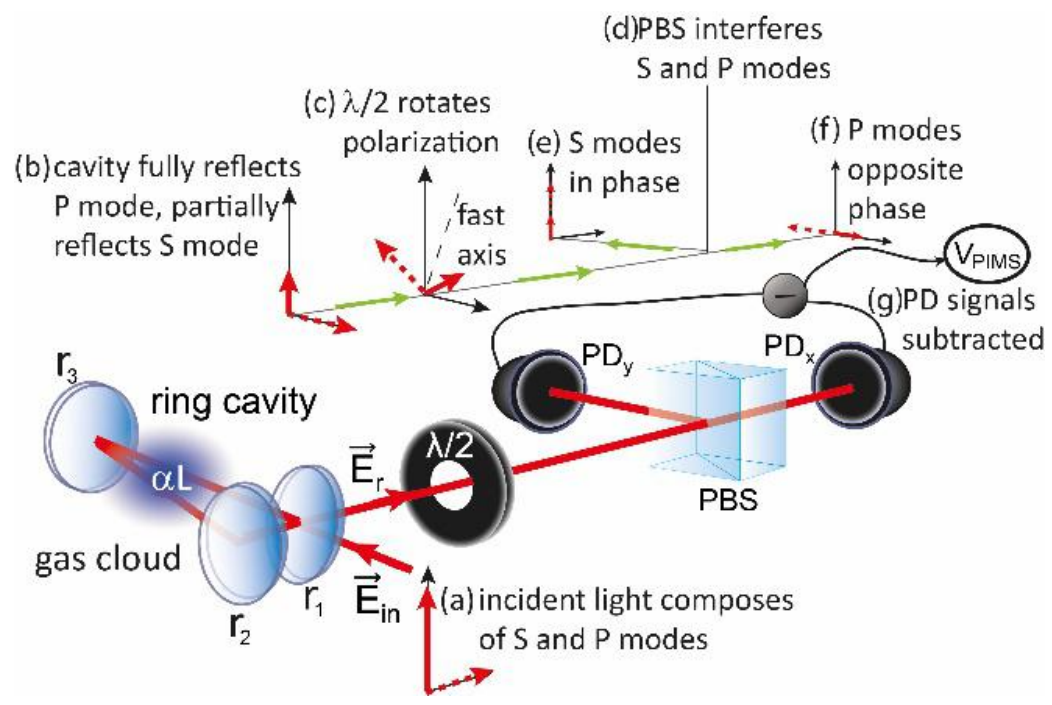

Fig. 1. Conceptual idea of PIMS system 
In order to extract the impedance matching state, and therefore the intra-cavity absorption, the orthogonally polarized probe and reference fields must be interfered using a polarization analyzer. The analyzer consists of a halfwave plate $(\lambda / 2)$ which is used to rotate the reflected light polarizations by 45 degrees (Fig. 1c), and also a diagonally aligned polarizing beam splitter (PBS) to facilitate the interference and project the fields onto different axes (Fig. 1d). As shown in Fig.1, the projections of the probe and reference field onto the vertical axis (y) are in phase and interfere constructively at the PBS reflection port (Fig. 1e). Whereas those at PBS transmission port are 180 degrees out of phase, resulting in destructive interference (Fig. 1f). The interference between the probe and reference beam can be visualized by taking the subtraction of the optical powers at the two PBS ports. The amplitude of this interference term is linearly proportional to the probe amplitude, and by extension, the intra-cavity absorption. This can be calibrated using a sample of known absorption.

In the experiment, we use a 1-meter-long impedance matched equilateral triangle cavity to minimize Rayleigh scattering effects and the Pound-Drever-Hall technique was applied to achieve frequency locking [4]. When away from the absorption transition, the probe beam reflected from the cavity is null [5] and the reference beam is subtracted out across the detector, leading to a zero background spectrum which is also first order immune to laser intensity fluctuations by common mode rejection.

\section{Results and Conclusion}

The experimental noise equivalent absorption shows a fractional absorption sensitivity of $3 \times 10^{-11} \mathrm{~Hz}^{-1 / 2}$, which is within a factor of 2 from the fundamental shot noise limit. While the current set of experiments have focused on trace detection of $12 \mathrm{C}$ in $\mathrm{CO}_{2}$, the ultra-high sensitivity afforded by the PIMS technique opens up the possibilities in measurement of other trace gas molecules with extremely low concentrations and various other interesting gas detection applications by moving to more exotic wavelengths such as mid-IR.

\section{References}

[1] J. Hodgkinson and R. P. Tatam, “Optical gas sensing: a review”, Meas. Sci. Technol., 24, 012004 (2013).

[2] H.-P. L. Gianluca Gagliardi, ed., Cavity-Enhanced Spectroscopy and Sensing, 0342-4111, Vol. 179 (Springer Berlin Heidelberg, 2014).

[3] J. H. Chow, I. M. C. Littler, D. S. Rabeling, D. E. McClelland, and M. B. Gray, Opt. Express 16, 7726 (2008).

[4] R. W. P. Drever, J. L. Hall, F. V. Kowalski, J. Hough, G. M. Ford, A. J. Munley, and H. Ward, Applied Physics B 31,97 (1983).

[5] J. Dong et al., "Optical cavity enhanced real-time absorption spectroscopy of CO2 using laser amplitude modulation”, Appl. Phys. Lett., 105, 053505 (2014). 